\title{
Rapid Spontaneous Resolution of Lumbar Intraspinal Facet Cyst after Lateral Lumbar Interbody Fusion
}

\author{
Shunsuke Fujibayashi ${ }^{1)}$, Bungo Otsuki ${ }^{1)}$, Shimei Tanida ${ }^{1)}$, Ryoichi Nagahara ${ }^{2)}$, Hideo Ito $^{3)}$ and Shuichi Matsuda ${ }^{1)}$ \\ 1) Department of Orthopedic Surgery, Graduate School of Medicine, Kyoto University, Kyoto, Japan \\ 2) Department of Orthopedic Surgery, Nagahama Red Cross Hospital, Nagahama, Japan \\ 3) Department of Orthopedic Surgery, Kyoto Shimogamo Hospital, Kyoto, Japan
}

\begin{abstract}
:
Introduction: Intraspinal facet cysts resistant to conservative treatment are treated surgically. Surgical treatment was generally resection and decompression, but complications of dural tear and recurrence sometimes occurred. We present good clinical results and rapid spontaneous resolution following treatment of five cases of lumbar intraspinal facet cyst after lateral lumbar interbody fusion (LLIF).

Methods: Multicenter series of five cases of lumbar intraspinal facet cyst with segmental instability treated with LLIF. The cross-sectional area (CSA) of the thecal sac and facet cyst on T2-weighted axial magnetic resonance imaging and the distance of facet joint (FJ) gap on axial computed tomography were measured preoperatively and postoperatively. Patient data and clinical and radiographic results were described.

Results: Of five patients, one was male and four were female, with an average age of 72.6 (61-76) years. The mean preoperative CSA of facet cyst was $40.09 \mathrm{~mm}^{2}$. In all cases, intraspinal facet cyst resolved within two weeks after LLIF and good clinical results were obtained. The mean CSA of the thecal sac increased from $64.18 \mathrm{~mm}^{2}$ preoperatively to 95.72 $\mathrm{mm}^{2}$ postoperatively. The mean distance of FJ gap increased from $0.8(0-1.5) \mathrm{mm}$ preoperatively to $3.1(0.5-6.0) \mathrm{mm}$ postoperatively.
\end{abstract}

Conclusions: LLIF may be indicated for intraspinal facet cysts with segmental instability. Keywords:

Intraspinal facet cyst, lateral lumbar interbody fusion (LLIF), indirect neural decompression, segmental instability

Spine Surg Relat Res 2020; 4(4): 328-332

dx.doi.org/10.22603/ssrr.2020-0084

\section{Introduction}

Intraspinal facet cysts have been increasingly reported in the literature because popular use of magnetic resonance imaging (MRI) has facilitated their diagnosis. They often cause symptoms such as claudication or radiculopathy with neural compression. Although the cause of intraspinal facet cysts remains unclear, it is likely related to instability and degenerative changes within the facet joints $(\mathrm{FJs})^{1-3)}$. Primary treatment is conservative, such as medications, steroid injections, and percutaneous rupture. If the cyst is resistant, resection and neural decompression are frequently applied. Recently, use of lateral lumbar interbody fusion (LLIF) in the treatment of lumbar degenerative disease as an alternative to conventional anterior or posterior procedures has been in- creasing, and the effects of indirect neural decompression were reported ${ }^{4-6)}$. The types of LLIF are extreme lateral interbody fusion (XLIF) and oblique lateral interbody fusion (OLIF). There are hitherto no reports about the use of LLIF in the treatment of lumbar intraspinal facet cysts. The report below describes good clinical results and rapid spontaneous resolution of lumbar intraspinal facet cysts after LLIF. Moreover, we evaluated the radiological effects and clinical outcomes.

\section{Materials and Methods}

Five patients with symptomatic intraspinal facet cyst were surgically treated at three institutions. Postoperative magnetic resonance imaging (MRI) and computed tomography 
Table 1. Summary of the Present Cases.

\begin{tabular}{|c|c|c|c|c|c|c|}
\hline Case No. & $\begin{array}{l}\text { Age (yrs), } \\
\text { Sex }\end{array}$ & Operative procedure & $\begin{array}{c}\text { Following } \\
\text { decompressive } \\
\text { surgery }\end{array}$ & \multicolumn{2}{|c|}{ Symptoms } & \\
\hline 1 & $61, \mathrm{~F}$ & OLIF (L4-L5)+PPS (L4-L5) & + & \multicolumn{2}{|c|}{ low back and leg pain } & \\
\hline 2 & $75, \mathrm{~F}$ & XLIF (L3-L5)+PPS (L3-L5) & + & \multicolumn{2}{|c|}{ low back and leg pain } & \\
\hline 3 & $76, \mathrm{~F}$ & OLIF (L4-L5)+PPS (L4-L5) & - & \multicolumn{2}{|c|}{ low back and leg pain } & \\
\hline 4 & $75, \mathrm{M}$ & OLIF (L3-L4)+PPS (L2-L5) & + & \multicolumn{2}{|c|}{ leg numbness } & \\
\hline 5 & $76, \mathrm{~F}$ & OLIF (L4-L5)+PPS (L4-L5) & - & \multicolumn{2}{|c|}{ leg pain } & \\
\hline $\begin{array}{l}\text { JOA score } \\
\text { (pre/post) }\end{array}$ & $\begin{array}{c}\text { Day of } \\
\text { postoperativ } \\
\text { MRI }\end{array}$ & $\begin{array}{c}\text { CSA of thecal sac } \\
\left(\mathrm{mm}^{2}\right) \\
(\text { pre/post })\end{array}$ & $\begin{array}{l}\text { SA of facet cyst } \\
\qquad\left(\mathrm{mm}^{2}\right) \\
(\text { pre })\end{array}$ & $\begin{array}{l}\text { Facet cyst } \\
\text { shrinkage }\end{array}$ & $\begin{array}{c}\text { Distance of FJ gap } \\
(\mathrm{mm}) \\
\text { (pre/post) }\end{array}$ & $\begin{array}{l}\text { Follow-up } \\
\text { (months) }\end{array}$ \\
\hline $18 / 28$ & 14 & $26.78 / 56.02$ & 15.94 & + & $1.4 / 3.7$ & 30 \\
\hline $22 / 27$ & 10 & $150.23 / 193.44$ & 76.65 & + & $0 / 6.0$ & 36 \\
\hline $16 / 20$ & 10 & $48.80 / 77.71$ & 44.11 & + & $0 / 4.1$ & 36 \\
\hline $20 / 22$ & 13 & $56.66 / 93.15$ & 42.35 & + & $1.5 / 2.0$ & 36 \\
\hline $19 / 25$ & 13 & $38.43 / 58.29$ & 21.39 & + & $1.2 / 3.6$ & 30 \\
\hline
\end{tabular}

OLIF indicates oblique lateral interbody fusion; XLIF, extreme lateral interbody fusion; PPS, percutaneous pedicle screw fixation; JOA, Japanese Orthopedic Association; CSA, cross-sectional area; FJ, facet joint

(CT) were obtained within two weeks after the surgery to determine the degree of indirect neural decompression and facet cyst. Clinical outcomes were evaluated by Japanese orthopedic Association (JOA) score (full score: 29 points) ${ }^{9}$.

\section{Measurement of CSA and FJ gap}

The cross-sectional area (CSA) of the thecal sac was measured both preoperatively and postoperatively using T2weighted axial MRI. CSA was measured using ImageJ software for image analysis (version 1.47; NIH, Bethesda, MD, USA). For MRI, a single axial slice through the center of the disc was used as the comparative measure location in axial views for measurement of the CSA of the thecal sac and facet cyst. The outline of the thecal sac in the selected axial view was traced manually, and the area enclosed $\left(\mathrm{mm}^{2}\right)$ was measured. FJ gaps were measured both preoperatively and postoperatively using an axial slice through the center of the disc in CT. The shortest gap of the FJ on the side of the cyst was defined as the FJ gap.

\section{Results}

Of five patients, one was male and four were female, with an average age of 72.6 (61-76) years. Two primary cases and three secondary cases following decompressive surgery for the same spinal level of facet cyst were included. XLIF was performed in one case and OLIF in four. The mean preoperative CSA of facet cyst was $40.09 \mathrm{~mm}^{2}$. In all cases, intraspinal facet cyst resolved within two weeks after LLIF and good clinical results were obtained. The mean JOA score increased from 19 points preoperatively to 24.4 points at final follow-up. The mean CSA of the thecal sac increased from $64.18 \mathrm{~mm}^{2}$ preoperatively to $95.72 \mathrm{~mm}^{2}$ postoperatively. The mean distance of the FJ gap increased from $0.8(0-1.5) \mathrm{mm}$ preoperatively to $3.1(0.5-6.0) \mathrm{mm}$ postop- eratively. The mean follow-up period was 33.6 (30-36) months. In all cases, bony union was achieved within one year and no recurrence of radicular symptoms was revealed throughout the follow-up period. The details of the patients' demographic data and results are described in Table 1.

\section{Case report 1}

A 61-year-old woman was presenting with complaint of low back pain and left leg pain prior to decompressive surgery for a right facet cyst at L4-L5 last year. X-ray showed mild instability at L4-L5 and MRI detected a facet cyst at L4-L5 on the left and fluid correction at the FJ, which indicated segmental instability (Fig. 1A, B). The patient was treated conservatively, but was not relieved from the symptoms. Direct resection of the facet cyst could result in neural injury because of the epidural adhesion following previous decompressive surgery. Our surgical strategy was preferential fixation for segmental instability, so we performed OLIF at L4-L5 with supplemental percutaneous pedicle screw fixation (PPS). In the event that the symptom did not improve postoperatively, our plan was to perform an additional posterior direct decompression for which the patient gave her informed consent. Pain relief was rapid postoperatively. At two weeks after the surgery, MRI revealed that remarkable indirect neural decompression was achieved and the $\mathrm{L}$ 4-L5 facet cyst resolved (Fig. 1C, D). The symptoms had alleviated significantly and JOA score improved from 18 to 28. MRI at one-year follow-up showed no recurrence of the facet cyst (Fig. 1E, F).

\section{Case report 2}

A 75-year-old woman underwent lumbar laminectomy from L2 to L5 at another hospital. Her low back pain and bilateral leg pain was deteriorated at three years after the surgery. X-ray showed segmental instability at L3-L4 and 

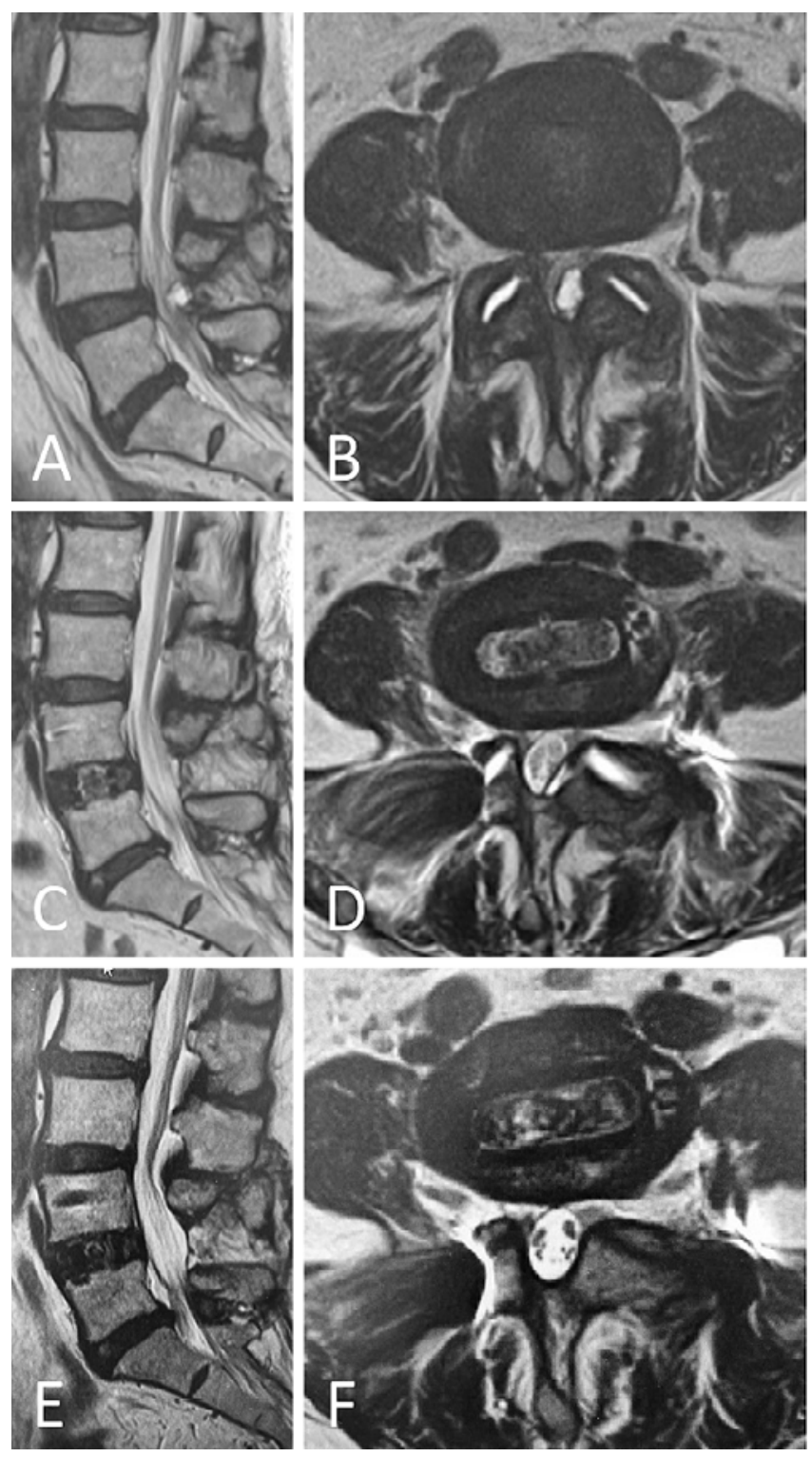

Figure 1. Case 1. Preoperative sagittal (A) and axial (B) T2-weighted MRI of the lumbar spine showing a facet cyst on the left at L4-L5 with compression of the neural elements and fluid correction at the FJ. Postoperative sagittal (C) and axial (D) T2-weighted MRI of the lumbar spine showing shrinkage of the previously noted facet cyst at L4-L5 and increase of fluid collection at the FJ. MRI at one-year follow-up showing no recurrence of facet cyst (E: sagittal, F: axial).

L4-L5 (Fig. 2A). MRI revealed a facet cyst at L3-L4 on the left, and severe spinal canal stenosis at L3-L4 and L4-L5 (Fig. 2B, C). We performed XLIF at L3-4 and L4-L5 with PPS (Fig. 2D). Her symptoms were resolved rapidly after the surgery. At 10 days after the surgery, MRI revealed indirect neural decompression and facet cyst shrinkage (Fig. 2E, F).

\section{Discussion}

Histopathologically, facet cyst has been referred to using various names, including ganglion cyst $^{10)}$, synovial cyst $^{10,11)}$ and pseudocystic lesion ${ }^{12)}$. In 1974, Kao et $\mathrm{al}^{13)}$ proposed the term "juxtafacet cyst" to represent both synovial and ganglion cysts. In 1995, Hsu et $\mathrm{al}^{17)}$ first used the term "intraspinal facet cyst" to designate cysts associated with the FJs regardless of whether synovial lining cells were present or not because there were cysts that exhibited the histological features of both synovial and ganglion cysts. The pathology and progression mechanism of facet cyst remain unclear. In 1996, Prestar et $\mathrm{al}^{18)}$ reported that rupture in the ligamentum flavum promoted the degeneration progressing to cyst formation by necrosis and fibrosis. Moreover, Kusakabe et al ${ }^{19)}$ clarified in a histologic and macroscopic study that facet cysts occur from rupture of the joint capsule in the ligamentum flavum where collagen fibers substitute for elastic fibers in areas where they become loose, and these then protrude into the spinal canal. Spinal instability also causes rupture and fissure in the ligamentum flavum, particularly in the capsular portion. In addition, the fissure develops into a cavity, mainly because of joint fluid from the connected FJ.

The first treatment option for intraspinal facet cyst is generally conservative, and consists of medications, steroid injection, or direct rupture under CT guidance. In the event that symptoms do not improve, surgical intervention is required. Generally, the surgical intervention is direct resection of the facet cyst. In some cases, however, direct resection of the facet cyst alone may be ineffective. Ikuta et $\mathrm{al}^{3)}$ reported that decompression alone may worsen instability with recurrence occurring in $8.6 \%$ of the patients. In our cases, cases 1,2 , and 4 underwent deterioration of segmental instability after decompressive surgeries. Case 4 was associated with diffuse idiopathic skeletal hyperostosis. Case 3 had degenerative disc change and foraminal stenosis with instability. Case 5 had degenerative spondylolisthesis with instability. Therefore, segmental instability is likely to play a significant role in the development of the facet cyst. Generally, in patients where instability is identified at the affected level, the addition of fusion may be indicated. Besides, facet cyst resection requires great labor due to complications such as dural tear and epidural venous plexus bleeding because of the epidural adhesion. Epstein ${ }^{20)}$ reported that the incidence of dural tear following the first decompressive operation was $3.8 \%$ and increased to $16.7 \%$ after the second operation. Scholz et $\mathrm{al}^{21)}$ reported that in $5.4 \%$ of patients, synovial cysts were not resected completely due to dural adhesions and high risk for dural tears.

Recently, LLIF has increased in popularity as treatment of lumbar degenerative disease. The procedure is associated with less invasiveness than conventional open decompression and fusion. Moreover, LLIF promotes indirect decompression on neural elements ${ }^{5,6)}$. However, the surgical indications for LLIF remain ambiguous. In general, LLIF is considered to be effective for mechanical low back pain caused by disc degeneration, mild canal stenosis with segmental instability, and degenerative scoliosis. It is thought that LLIF cannot adequately address pathologies such as an uncontained-type herniated nucleus pulposus, facet hyperostosis, and so on. 

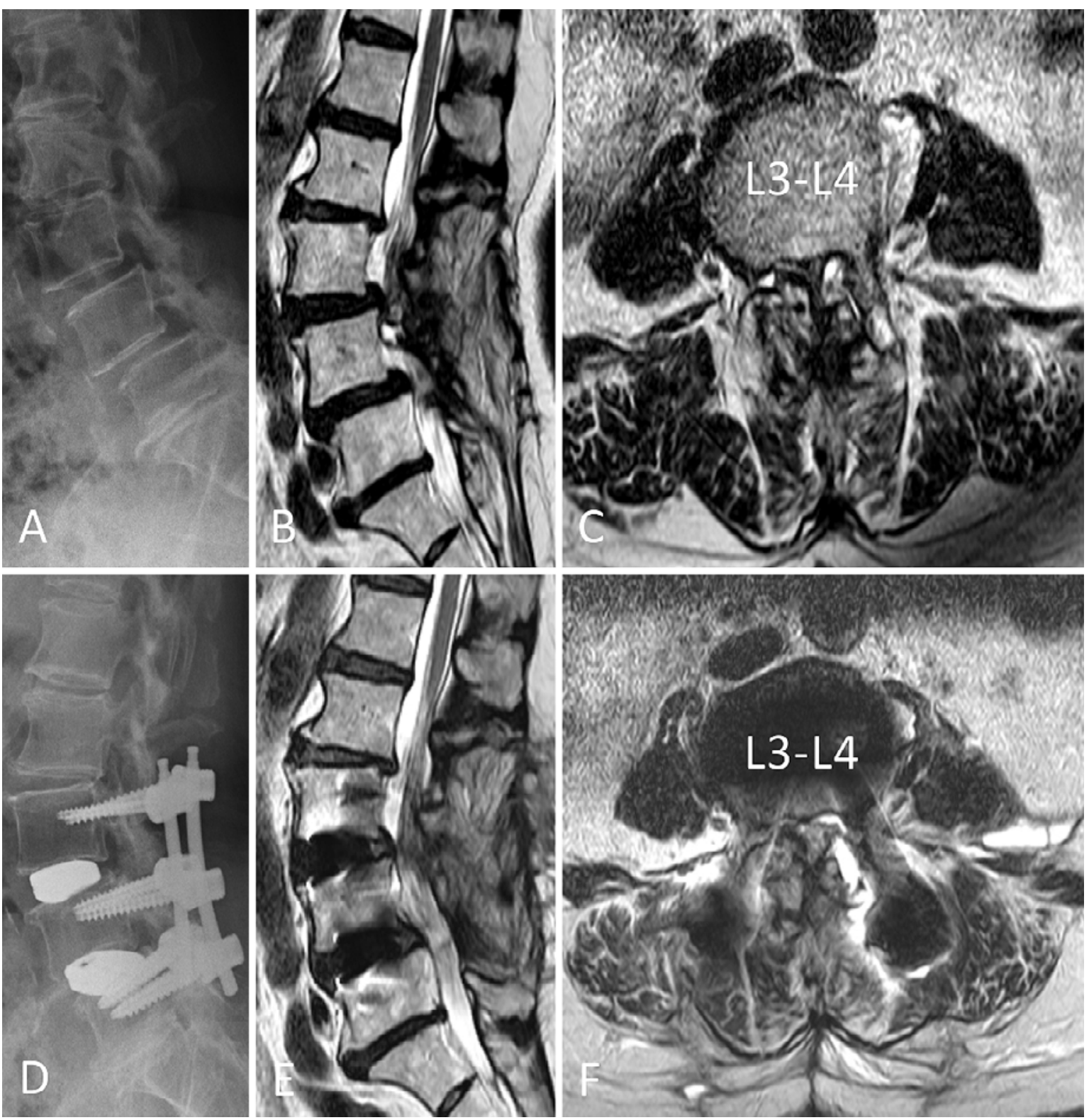

Figure 2. Case 2. Preoperative lateral X-ray (A) demonstrating segmental instability at L3-L4 and L4-L5. Preoperative sagittal (B) and axial (C) T2-weighted MRI showing a facet cyst on the left at L3-L4 with compression of the neural elements. Lateral X-ray after two-level XLIF (D). Postoperative sagittal (D) and axial (E) T2-weighted MRI showing shrinkage of facet cyst and indirect neural decompression.

LLIF is not considered as indicated for lumbar facet cysts up to date ${ }^{5}$. However, the five cases presented here produced good results and the facet cysts resolved spontaneously without direct resection. These results suggest that LLIF can be indicated for facet cysts with segmental instability, especially for secondary case following decompressive surgery. However, the reason why facet cysts resolve soon after LLIF is unknown. One of the reasons may be stabilization of the affected level after LLIF. This, however, cannot fully explain the rapid spontaneous resolution of the cysts. Another reason may be due to extension of the FJ and ligamentum flavum through LLIF. In fact, FJ gap was found to spread significantly postoperatively than preoperatively in all cases. We measured the difference between preoperative and postoperative distance of the FJ gap at the affected level in CT. In- creases in the distance were $2.3 \mathrm{~mm}$ in Case $1,6.0 \mathrm{~mm}$ in Case 2, $4.1 \mathrm{~mm}$ in Case 3, $0.5 \mathrm{~mm}$ in Case 4, and $2.4 \mathrm{~mm}$ in Case 5. Simultaneously, MRI demonstrated that facet fluid collection increased more postoperatively than preoperatively (Fig. 1B, D). We speculate that when the FJ gap is spread by LLIF, negative pressure builds up within the FJ, and the fluid in the cyst flows into the FJ. Therefore, the cyst may shrink due to the increase in FJ fluid.

The limitation of this study is the fact that FJ arthrography was not performed for all the cases. This makes it unclear as to whether the intraspinal facet cyst communicates with the FJ. 


\section{Conclusions}

Five cases of intraspinal facet cyst resolved rapidly after LLIF and good clinical results were obtained. The results suggest that LLIF can be indicated for intraspinal facets cyst with instability.

Conflicts of Interest: The authors declare that there are no relevant conflicts of interest.

Ethical Approval: This study was performed with the approval of the institutional ethics committee of Kyoto University (R1955).

Author Contributions: Conceived and designed the study: SF; analyzed the data: SF; collected the data and read the radiograph: $\mathrm{SF}, \mathrm{BO}, \mathrm{ST}, \mathrm{RN}, \mathrm{HI}$; wrote the paper: SF; supervision of the manuscript: SM; and all authors read and approved the final manuscript.

Informed Consent: Informed consent was obtained from all participants in this study.

\section{References}

1. Daentzer D, Studer D. Thoracic juxtafacet cyst (JFC) as a rare cause of myelopathy-an additional reference to support the instability theory. Joint Bone Spine. 2010;77(2):178-80.

2. Martha JF, Swaim B, Wang DA, et al. Outcome of percutaneous rupture of lumbar synovial cysts: a case series of 101 patients. Spine J. 2009;9(11):899-904.

3. Ikuta K, Tono O, Oga M. Prevalence and clinical features of intraspinal facet cysts after decompression surgery for lumbar spinal stenosis. J Neurosurg Spine. 2009;10(6):617-22.

4. Ozgur BM, Aryan HE, Pimenta L, et al. Extreme lateral interbody fusion (XLIF): a novel surgical technique for anterior lumbar interbody fusion. Spine J. 2006;6(4):435-43.

5. Fujibayashi S, Hynes RA, Otsuki B, et al. Effect of indirect neural decompression through oblique lateral interbody fusion for degenerative lumbar disease. Spine. 2015;40(3):E175-82.

6. Oliveira L, Marchi L, Coutinho E, et al. A radiographic assessment of the ability of the extreme lateral interbody fusion procedure to indirectly decompress the neural elements. Spine. 2010;35 (26 Suppl):S331-7.

7. Morr S, Kanter AS. Complex regional pain syndrome following lateral lumbar interbody fusion. Case report. J Neurosurg Spine. 2013;19(4):502-6.

8. Ahmadian A, Deukmedjian AR, Abel N, et al. Analysis of lumbar plexopathies and nerve injury after lateral retroperitoneal transpsoas approach: diagnostic standardization. J Neurosurg Spine. 2013;18(3):289-97.

9. Fujiwara A, Kobayashi N, Saiki K, et al. Association of the Japanese Orthopaedic Association score with the Oswestry Disability Index, Roland-Morris Disability Questionnaire, and Short-Form 36. Spine. 2003;28(14):1601-7.

10. Bhushan C, Hodges FJ, Wityk JJ. Synovial cyst (ganglion) of the lumbar spine simulating extradural mass. Neuroradiology. 1979;18 (5):263-8.

11. Boviatsis EJ, Staurinou LC, Kouvialis AT, et al. Spinal synovial cysts: pathogenesis, diagnosis and surgical treatment in a series of seven cases and literature review. Eur Spine J. 2008;17(6):831-7.

12. Tsuji H, Handa N, Handa O, et al. Postlaminectomy ossified extradural pseudocyst. J Neurosurg Spine. 1990;73(5):785-7.

13. Kao CC, Winkler SS, Turner JH. Synovial cyst of spinal facet. Case report. J Neurosurg. 1974;41(3):372-6.

14. Banning CS, Thorell WE, Leibrock LG. Patient outcome after resection of lumbar juxtafacet cysts. Spine. 2001;26(8):969-72.

15. Lyons MK, Atkinson JL, Wharen RE, et al. Surgical evaluation and management of lumbar synovial cysts: The Mayo Clinic experience. J Neurosurg. 2000;93(1 Suppl):53-7.

16. Maezawa $Y$, Baba $H$, Uchida $K$, et al. Spontaneous remission of a solitary intraspinal synovial cyst of the lumbar spine. Eur Spine J. 2000;9(1):85-7.

17. Hsu KY, Zucherman JF, Shea WJ, et al. Lumbar intraspinal synovial and ganglion cysts (facet cysts). Ten-year experience in evaluation and treatment. Spine. 1995;20(1):80-9.

18. Prestar FJ. Juxta facet cysts of the lumbar spine. Minim Invasive Neurosurg. 1996;39(2):45-9.

19. Kusakabe T, Kasama F, Aizawa T, et al. Facet cyst in the lumbar spine: radiological and histopathological findings and possible pathogenesis. J Neurosurg Spine. 2006;5(5):398-403.

20. Epstein NE. Lumbar laminectomy for the resection of synovial cysts and coexisting lumbar spinal stenosis or degenerative spondylolisthesis: an outcome study. Spine. 2004;29(9):1049-55.

21. Scholz C, Hubbe U, Kogias E, et al. Incomplete resection of lumbar synovial cysts - Evaluating the risk of recurrence. Clin Neurol Neurosurg. 2015;136:29-32.

Spine Surgery and Related Research is an Open Access journal distributed under the Creative Commons Attribution-NonCommercial-NoDerivatives 4.0 International License. To view the details of this license, please visit (https://creativeco mmons.org/licenses/by-nc-nd/4.0/). 